\title{
Ultrafast two-bit all-optical analog-to-digital conversion based on femtosecond soliton sequence sampling.
}

\begin{abstract}
Realization of two-bit all-optical analog-to-digital conversion for an analog signal sampled by a femtosecond soliton sequence is investigated. Two approaches are suggested. The first one is based on filtering the broadened soliton spectrum after evolution over half of the soliton period in a standard single-mode fiber. In the second approach, the pulse is temporally sampled at the specified times after propagating through one soliton period. The sampled soliton sequence must be amplified to achieve an initial peak power of between 0 and $75 \mathrm{~kW}$ for the first method and between 0 and $66 \mathrm{~kW}$ for the second method. The soliton pulse-width is $50 \mathrm{fs}$. Based on the resulted peak power, the " 0 " or " 1 " bit is generated with reference to the threshold values. Subsequently, the digital gray code is produced at the outputs. The effect of inaccuracy in filter frequency and fiber length are also studied in this paper. The first method is sensitive to variations in the filter frequency, whereas the second method is affected by the fiber length inaccuracy.
\end{abstract}

Keyword: Ultrafast two-bit; Analog-to-digital conversion; Femtosecond soliton sequence sampling. 